\title{
What have we learnt from 40 years of research on Intensive Case Management?
}

\author{
MAX MARSHALL
}

\begin{abstract}
The aims of the Editorial are to summarise what we know for certain from clinical trials of Intensive Case Management, and to highlight lessons for clinicians and researchers. I will upon two systematic reviews of trials of Intensive Case Management versus standard care or low intensity case management. Both incorporated a meta-regression which examined the effect of fidelity to the Assertive Community Treatment model on outcome. The effectiveness of Intensive Case Management was limited to improving patient satisfaction and reducing attrition. Intensive Case Management teams organised according to the Assertive Community Treatment model offered the additional benefit of reducing days in hospital, but only when the team's clients had been high users of hospital care over the previous 12 months. Four important lessons can be drawn: a) Changes to the process of care tend to affect process variables, not outcome variables. b) Complex interventions must be defined meticulously in clear terminology. c) Researchers must demonstrate that complex interventions have been properly implemented in clinical trials. d) It is important to remember that in a clinical trial a successful outcome is determined as much by the control group as by the intervention.
\end{abstract}

\section{INTRODUCTION}

Clinical trials of case management have been taking place since the early 1970 s, and I have been analysing their findings for almost 20 years. In that time I have learnt that authors who do not define their chosen terminology are in danger of confounding their readership, leaving them more ignorant of the subject than before they read the article. Hence, risking dullness, I will begin by defining the terms I intend to use throughout, namely:

Case management - an approach to community care in which each severely mentally ill person is allocated a community worker (i.e. case manager) who takes primary responsibility for their welfare. As a minimum this responsibility includes: keeping contact with the person, assessing their needs, and ensuring that these needs are met.

Intensive Case Management (ICM) - the case managers carry small caseloads, usually considerably less than twenty clients per case manager. Intensive case managers are normally clinicians who act as therapists in addition to their case management duties.

Address for correspondence: Professor M. Marshall, The Lantern Centre, Vicarage Lane, Fulwood, Preston. PR2 8DY (United Kingdom).

E-mail: max.marshall@manchester.ac.uk

Declaration of Interest: The reviews described in this editorial were funded by a grant from the Department of Health Policy Research Programme. I have not received any honoraria or grants from drug companies, lectures or consultancies, over the past two years.
Assertive Community Treatment (ACT) - a carefully specified form of intensive case management, defined by treatment manuals and fidelity scales. It includes such special features as: daily team meetings, case sharing (where several team members work with each client), 24 hour availability, and doctors as full team members.

Over the past twenty years, the progress of community psychiatry could be described as the "Triumph of Case Management". Most modern healthcare systems now incorporate some form of case management for severely mentally ill people, often of an intensive nature. Thus ACT programs have been set up across the US, Canada, Australia and Europe (Bond et al., 2001; Burns et al., 2001). In England, for example, the National Service Framework has mandated the setting up of one hundred and seventy high-fidelity Assertive Outreach teams (Department of Health, 1999).

It is a shame that this development has not been accompanied by an improving evidence base, but sadly this is not so. As Intensive Case Management has become standard practice around the globe, the evidence base on which it is grounded has looked increasingly fragile. The purpose of this editorial is to summarise what we know for certain about case management after 40 years of research and many clinical trials, and to highlight what we may learn as clinicians and researchers.

\section{A HISTORY OF TWO REVIEWS}

In 2002 Professor Tom Burns and I were commissioned by the English Department of Health to carry out 
a new systematic review of Intensive Case Management. The Department was concerned by the surprisingly unpromising findings of UK trials in this area, and so the review aimed to understand why trials of apparently similar styles of Intensive Case Management were having contradictory results. In particular we set out to test two theories:

1) Intensive Case Management is only effective when it adheres to the principles of Assertive Community Treatment;

2) The inconsistent findings between clinical trials of intensive case management can be explained by differences in the trial control groups.

The control group factors that interested us were:

a) whether the trial had taken place in the United States;

b) the degree of low intensity case management in the control group; and

c) the year in which the study had taken place.

In other words, we were testing whether intensive case management only worked in the US (perhaps because of the fragmented nature of health care in that country), and whether it had become less effective as "control" conditions had shifted towards more case management-like models.

On completion of this review we thought we knew the answers to the case management conundrum, but our opinions were only partially confirmed by the findings of a new UK clinical trial. So we returned to the drawing board and completed an updated analysis, focussing on reductions in hospital user alone and featuring a new confounding variable and analytical method. Finally I believe we arrived at an accurate understanding of the complex reality contained with the trial data.

\section{METHOD AND FINDINGS OF THE FIRST REVIEW}

For our initial review we used a systematic search strategy to identify randomised controlled trials that compared Intensive Case Management (including Assertive Community Treatment) against either 'standard care' or low intensity case management, for people with severe mental illness. We defined 'standard care' as care from either a community mental health team or an out-patient clinic, and 'low intensity case management' as case management where the caseload was greater than 20 . We excluded trials where the control intervention was: hospi- tal admission, remaining in hospital; or some other form of Intensive Case Management.

Information on the primary outcome measures (attrition, hospital use, clinical and social outcomes) were extracted following established Cochrane methodology (Higgins et al., 2005). For every included trial we made a rating of fidelity of case management practice to the Assertive Community Treatment model. For this we used the IFACT scale, which has subscales for team composition and team organisation (McGrew et al., 1994). We based this rating on information from published papers and data obtained directly from trialists.

We knew from our conversations with trialists that "multi-centre" trials often struggled to implement the same model of case management in each trial site. Hence, where possible, we treated individual sites in multi-centre studies as separate centres. To do this it was often necessary to obtain original patient data from trialists so that centre data could be disaggregated. Our data analysis strategy was as follows:

a) We identified outcomes where there were sufficient data to be sure that intensive case management had no effect on the outcome.

b) We identified outcomes where wide confidence intervals indicated that insufficient data were available to judge the effectiveness of Intensive Case Management.

c) We identified outcomes where intensive case management clearly had a consistent effect across trials.

d) We identified outcomes where statistical heterogeneity was present between the findings of different trials. When data from sufficient trials were available, we then explored the reasons for this "heterogeneity" using a statistical technique known as "meta-regression", which allowed us to determine if the discrepancy could be explained by: differences in the fidelity of the intervention to the ACT model (IFACT score); the degree of case management in the control service; the year the study was conducted; and the location of the study (US versus non-US). Thus we were able to test the key theories that had been put forward to explain the inconsistency between trials.

Our search found 1199 abstracts of which 356 were duplicates. We requested 328 papers which yielded 126 potentially eligible trials. Of these 88 were excluded after closer scrutiny, leaving 38 included trials, 4 of which had no useable data. Of the 34 trials providing data: twentyone trials were from the United States $(n=4290)$; eleven were from the $E U(n=2340)$ of which eight were from the UK $(n=1676)$; and two $(n=196)$ were from elsewhere 
(Canada and Australia). The mean age of participants in the trials was 38.2 years, $34.8 \%$ were female, $63.2 \%$ had schizophrenia or schizophrenia-like disorder, and $38.1 \%$ were from black ethnic minorities (although this may be an overestimate as only 23 of 34 trials provided information on this variable). Individual patient data were obtained for 4 multi-centre trials which were disaggregated into 23 'single site' trials.

Perhaps the most surprising finding of our analysis was the number of outcomes on which Intensive Case Management definitely had no clinically significant effect, irrespective of fidelity to the Assertive Community Treatment model. These outcomes included: quality of life, negative symptoms, social functioning, depression, alcohol use, and drug abuse. There were insufficient data to draw conclusions on the effect of Intensive Case Management on homelessness, death, suicide, imprisonment, arrest, or compliance with treatment.

However, Intensive Case Management was consistently better than control at improving patient satisfaction, though there was no evidence to suggest that teams adhering to the Assertive Community Treatment model were better at this than those that did not.

On three variables: total symptoms; attrition rates; and use of hospital care, we found evidence of statistical heterogeneity, and so carried out a meta-regression analysis. For total symptoms were unable to find the source of heterogeneity between trials, perhaps it is just to do with the fact that some teams have better psychiatrists than others. For attrition rates, we found, to our surprise, that teams adhering to the Assertive Community Treatment model were slightly less effective at maintaining contact with patients than other Intensive Case Management teams (though both were better than control). We found that Intensive Case Management teams with greater fidelity to the Assertive Community Treatment model, (especially on the IFACT subscale that measured team organisation) were effective at reducing days in hospital, whereas those with low fidelity, were not. In other words to reduce hospital use, an intensive case management team needed to have: daily team meetings, 24 hour cover at evenings and weekends, team management practices, a team leader who carried a case load, and a "no discharge" policy.

\section{BACK TO THE DRAWING BOARD}

On completion of our review, in 2004, Tom and I were invited to present our findings at a Department of Health seminar on new research on Assertive Outreach. The seminar also included an initial presentation of the find- ings of the REACT study, which was the first UK randomised controlled trial to practice Assertive Community Treatment according to strict fidelity criteria (Killaspy $e t$ al., 2006). We were gratified to find that the trial reported superiority in satisfaction and attrition rates for the intervention, but we were astounded to find that the trial found no effect on days in hospital, despite close adherence to the Assertive Community Treatment model. It was obvious that we had overlooked something in our analysis.

After discussion with our statistician, Chris Roberts, of the University of Manchester, we decided to embark on a new analysis focussing entirely on the outcome of hospital use (Burns et al., 2007). We decided to test the intriguing suggestion that Intensive Case Management was effective only against a background of high reliance on hospital care. We had previously avoided this analysis as it presented difficult technical challenges, due to the fact that the best measure of background hospital use was control group admission rates, which was unfortunately confounded with the difference in hospital use between the treatment and control groups. We got around this problem by returning to the original data sources and trialists, to establish the participants' use of hospital care in the twelve months before entering the trial. We then included this variable in a meta-regression of days in hospital, in addition to our previous co-variates, which included fidelity to the Assertive Community Treatment Model. Obtaining these data was a lengthy process, so we also decided to update the review and include any new studies identified in the analysis. We identified 29 eligible trials providing data on mean days per month in hospital. The mean age of participants was 37.9 years, $37 \%$ were female, $66 \%$ had schizophrenia or schizophrenialike disorder, and $37 \%$ were from ethnic minorities. The mean attrition rate was $4 \%$.

After subdividing multi-centre trials into individual sites we obtained a total of 52 centres for analysis. Thirty five centres were from the US (3271 patients); 15 were from the European Union (2494 patients), including 13 from the UK ( $n=1541$ patients); and two ( $n=196$ patients) were from elsewhere (Canada and Australia). For 42 of these sites we obtained data on use of inpatient care in the 12 months before the trial began. Our meta-regression showed a strong correlation between the ability of intensive case management to reduce hospital use and the underlying level of hospital use. Thus the treatment effect was estimated to decrease by 0.31 bed days for each mean bed day difference between sites. Whilst fidelity to the organisation scale of the IFACT scale still had an effect, this was much reduced from our previous analysis. 


\section{CONCLUSION}

In summary we found that the effectiveness of Intensive Case Management was pretty much limited to improving patient satisfaction and reducing attrition. Intensive Case Management teams that were organised according to the Assertive Community Treatment model offered the additional benefit of reducing days in hospital, but only under circumstances where the team's clients had been high users of hospital care over the previous 12 months. We were unable to say whether this was due to the characteristics of the clients or the characteristics of their local mental health services, though we suspect it is largely due to the latter. In other words, Assertive Outreach Teams find it fairly easy to reduce the use of hospital care, when it is grossly over used, but find much harder to reduce hospital care when it is being used parsimoniously.

So from nearly 40 years of clinical trials of case management, I would draw the following lessons for clinicians and researchers:

1) Changes to the process of care tend to affect process variables, not outcome variables. It was naive to believe that simply retaining a patient in the community and providing them with a case manager would actually affect core aspects of the disease process, such as symptoms or functioning. By altering the process of care, Intensive Case Management improved process variables, such as satisfaction, attrition, and hospital use, but it had little impact on outcome. In future we need to look more to the therapeutic content of interventions, rather than purely structural considerations.

2) Complex interventions must be defined meticulously in clear terminology or confusion will result. Much of my effort over the past 20 years has been expended trying to work out what trialists meant when they used terms like "intensive case management", "assertive outreach" or similar terms from a myriad of synonyms. So great was the resulting confusion that a whole corpus of medical research was rendered almost useless. Journal editors and funding bodies should insist that authors use clear and consistent terminology when describing complex interventions.

3) Trialists must adhere to the definitions of complex interventions and demonstrate that the intervention has been properly implemented. I think it is fair to say that many trialists embarked on studies of Intensive Case Management, without a clear idea of what they were trying to evaluate, hence the phenomenon of multi-centre trials that implemented different interventions in different participating sites. Fundamentally this problem could be attributed to confusing terminology and the lack of fidelity scales. In future, the developers of complex interventions should be obliged to provide fidelity scales.

4) In a clinical trial a successful outcome is determined as much by the control group as by the intervention. Meta-analysts often overlook the fact that the outcome of a trial is partly determined by the level of performance of the control group. When heterogeneity is present in a meta-analysis, particularly one involving trials of complex interventions, we should suspect differences between control groups.

\section{REFERENCES}

Bond G., Drake R., Mueser K. \& Latimer E. (2001). Assertive community treatment for people with severe mental illness: critical ingredients and impact on patients. Disease Management and Health Outcomes 9, 141-159.

Burns T., Fioritti A., Holloway F., Malm U. \& Rossler W (2001). Case management and assertive community treatment in Europe. Psychiatric Services 52, 631-636.

Burns T., Catty J., Lockwood L., Roberts C. \& Marshall M. (2007). Use of intensive case management to reduce time in hospital in people with severe mental illness: systematic review and meta-regression. British Medical Journal Aug 18, 335(7615)-336.

Department of Health. (1999). A National Service Framework for Mental Health. Department of Health: London.

Higgins J. \& Green S. (Eds.) (2005). Cochrane Handbook for Systematic Review's of Interventions 4.2.5 [updated May 2005]. Wiley \& Sons, Ltd: Chichester (UK).

Killaspy H., Bebbington P., Blizard R., Johnson S., Nolan F., Pilling S. \& King M. (2006). The REACT study: randomised evaluation of assertive community treatment in north London. British Medical Journal 332, 815-820.

McGrew J., Bond G., Dietzen L. \& Salyers M. (1994). Measuring the fidelity of implementation of a mental health program model. Journal of Consulting and Clinical Psychology 62, 670-678. 